\title{
Intraplaque Hemorrhage and the Plaque Surface in Carotid Atherosclerosis: The Plaque At RISK Study (PARISK)
}

\author{
A.C. van Dijk, M.T.B. Truijman, B. Hussain, T. Zadi, G. Saiedie, A.A.J. de Rotte, M.I. Liem, A.F.W. van der Steen, M.J.A.P. Daemen,
} P.J. Koudstaal, P.J. Nederkoorn, J. Hendrikse, (DM.E. Kooi, and A. van der Lugt

\begin{abstract}
BACKGROUND AND PURPOSE: An important characteristic of vulnerable plaque, intraplaque hemorrhage, may predict plaque rupture. Plaque rupture can be visible on noninvasive imaging as a disruption of the plaque surface. We investigated the association between intraplaque hemorrhage and disruption of the plaque surface.
\end{abstract}

MATERIALS AND METHODS: We selected the first 100 patients of the Plaque At RISK study, an ongoing prospective noninvasive plaque imaging study in patients with mild-to-moderate atherosclerotic lesions in the carotid artery. In carotid artery plaques, disruption of the plaque surface (defined as ulcerated plaques and/or fissured fibrous cap) and intraplaque hemorrhage were assessed by using MDCTA and 3T MR imaging, respectively. We used a $\chi^{2}$ test and multivariable logistic regression to assess the association between intraplaque hemorrhage and disrupted plaque surface.

RESULTS: One hundred forty-nine carotid arteries in 78 patients could be used for the current analyses. Intraplaque hemorrhage and plaque ulcerations were more prevalent in symptomatic compared with contralateral vessels (hemorrhage, 38\% versus $11 \%$; $P<.001$; and ulcerations, $27 \%$ versus $7 \% ; P=.001)$. Fissured fibrous cap was more prevalent in symptomatic compared with contralateral vessels $(13 \%$ versus $4 \% ; P=.06$ ). After adjustment for age, sex, diabetes mellitus, and degree of stenosis, intraplaque hemorrhage was associated with disrupted plaque surface (OR, 3.13; $95 \% \mathrm{Cl}, 1.25-7.84)$ in all vessels.

CONCLUSIONS: Intraplaque hemorrhage is associated with disruption of the plaque surface in patients with a carotid artery stenosis of $<70 \%$. Serial studies are needed to investigate whether intraplaque hemorrhage indeed increases the risk of plaque rupture and subsequent ischemic stroke during follow-up.

ABBREVIATIONS: ECST = European Carotid Surgery Trial; PARISK = Plaque At RISK

$\mathbf{T}$ he need to identify patients with mild-to-moderate carotid artery stenosis and an increased stroke risk who might benefit from surgical treatment has shifted research interest from assess-

Received December 23, 2014; accepted after revision March 14, 2015. From the Departments of Radiology (A.C.v.D., B.H., T.Z.,G.S., A.v.d.L.), Neurology (A.C.v.D., P.J.K.), and Biomedical Engineering (A.F.W.v.d.S.), Erasmus Medical Center Rotterdam, the Netherlands; Departments of Radiology (M.T.B.T., M.E.K.) and Clinical Neurophysiology (M.T.B.T.) and Cardiovascular Research Institute Maastricht School for Cardiovascular Diseases (M.T.B.T., M.E.K.), Maastricht University Medical Center, Maastricht, the Netherlands; Department of Radiology (A.A.J.d.R., J.H.), University Medical Center Utrecht, Utrecht, the Netherlands; and Departments of Neurology (M.I.L., P.J.N.) and Pathology (M.J.A.P.D.), Amsterdam Medical Center, Amsterdam, the Netherlands.

This work was performed within the framework of the Center for Translational Molecular Medicine (www.ctmm.nl), project PARISK (grant 01C-202), and was supported by the Dutch Heart Foundation.

Paper previously presented in part at: Annual Meetings of the European Congress of Radiology, May 28-31, 2013, Vienna, Austria; the European Stroke Conference, May 28-31, 2013, London, UK; and the European Society for Magnetic Resonance in Medicine and Biology, October 3-5, 2013, Toulouse, France. ment of the degree of carotid stenosis to assessment of vulnerable plaque characteristics. ${ }^{1}$ Vulnerable plaques are atherosclerotic plaques more prone to rupture and are associated with a higher risk for thromboembolism and ischemic stroke. ${ }^{2,3}$ Intraplaque hemorrhage is an important characteristic of the vulnerable plaque. ${ }^{4}$ Prevalence of intraplaque hemorrhage has been shown to be higher in symptomatic than in asymptomatic lesions. ${ }^{5}$ Moreover, the presence of intraplaque hemorrhage in carotid artery disease is associated with an increased risk of cerebral ischemic events. $^{6-8}$

The pathophysiologic mechanism leading to intraplaque hemorrhage is a topic of debate. However, a common viewpoint is that small leaky neovessels in the atherosclerotic plaques are a likely

Please address correspondence to Aad van der Lugt, MD, PhD, Erasmus MC-University Medical Center Rotterdam, Department of Radiology, 's Gravendijkwal 230, 3015 CE Rotterdam, the Netherlands; e-mail: a.vanderlugt@erasmusmc.nl

三 Indicates article with supplemental on-line tables.

http://dx.doi.org/10.3174/ajnr.A4414 
source of intraplaque hemorrhage. ${ }^{5,9,10}$ The presence of intraplaque hemorrhage is thought to initiate several biologic processes like phagocytosis and local inflammation, leading to the release of proteolytic enzymes, deposition of free cholesterol and subsequently plaque growth, plaque destabilization, and possible plaque rupture. ${ }^{5,9-12}$ Plaque rupture can be visible on imaging as a disruption of the atherosclerotic plaque surface (plaque ulceration and/or a fissured fibrous cap). ${ }^{13,14} \mathrm{~A}$ previous study reported that plaque ulceration on CTA was useful for the prediction of intraplaque hemorrhage on MR imaging in a broad group of symptomatic patients referred for carotid artery imaging. ${ }^{15} \mathrm{Ul}-$ cerated plaques themselves are independently associated with an increased risk of ipsilateral ischemic events as well. ${ }^{16,17}$

The aim of the current study was to investigate the association between intraplaque hemorrhage, as assessed on MR imaging, and disruption of the plaque surface, assessed on MDCTA, in symptomatic patients with a carotid artery stenosis of $<70 \%$.

\section{MATERIALS AND METHODS \\ Study Population}

Patients were derived from the Plaque At RISK (PARISK) study (clinical trials.gov, NCT01208025). Details of the PARISK study are previously described. ${ }^{18}$ The PARISK study is an ongoing prospective multicenter cohort study focusing on the identification of patients with mild-to-moderate carotid artery stenosis with an increased risk of recurrent stroke by using noninvasive plaque imaging. Eligible for inclusion are patients with a TIA, including amaurosis fugax, or minor stroke in the carotid artery territory and a mild-to-moderate stenosis $(30 \%-69 \%)$ of the ipsilateral internal carotid artery. "TIA" was defined as an episode of temporary and focal cerebral dysfunction of vascular origin, lasting for a maximum 24 hours, leaving no persistent neurologic deficits. "Minor stroke" was defined as an episode of temporary and focal cerebral dysfunction of vascular origin, lasting for $>24$ hours or a nondisabling stroke with a modified Rankin Scale score of $\leq 3$. "Amaurosis fugax" was defined as a sudden loss of vision of presumed vascular origin and confined to 1 eye. The degree of stenosis was determined with clinically obtained Doppler sonography or MDCTA. The upper cutoff value of $70 \%$ was based on the NASCET criteria. The lower cutoff value was an atherosclerotic plaque with a thickness of at least 2-3 mm, which corresponds to a European Carotid Surgery Trial (ECST) stenosis of $30 \% .{ }^{19}$ Exclusion criteria were a probable cardiac source of embolism, a clotting disorder, severe comorbidity, standard contraindications for MR imaging, a documented allergy to MR imaging or CT contrast agents, or a renal clearance of $<30 \mathrm{~mL} / \mathrm{min}$. Institutional review board approval was obtained in all university hospitals, and all patients gave written informed consent. For the current analyses, we selected the first 100 included patients.

\section{Cardiovascular Risk Factors}

"Hypercholesterolemia" was defined as fasting total cholesterol of $>5 \mathrm{mmol} / \mathrm{L}$ or the use of cholesterol-lowering medication at the time of the TIA or ischemic stroke. We defined "hypertension" as systolic blood pressure of $>140 \mathrm{~mm} \mathrm{Hg}$ or a diastolic blood pressure of $>90 \mathrm{~mm} \mathrm{Hg}$ during 2 episodes of at least 15 minutes of continuous noninvasive blood pressure measurement or treat-

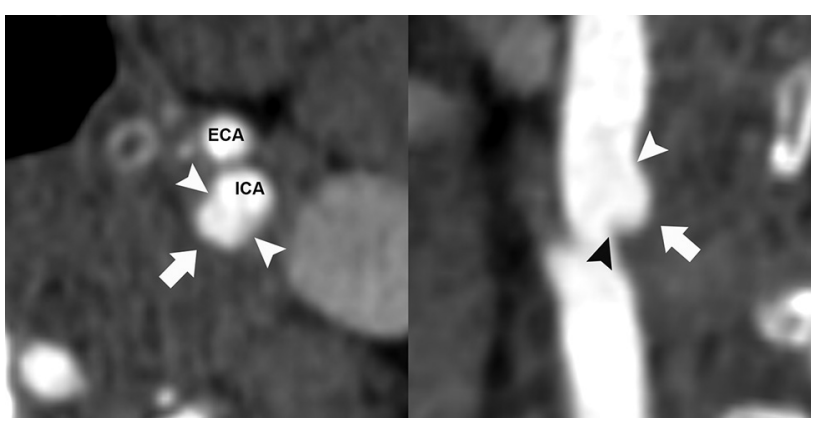

FIG 1. Plaque ulceration on MDCTA. Transversal (left) and longitudinal (right) MDCTA images of the carotid bifurcation. A "plaque ulceration," defined as the extension of contrast material in the atherosclerotic plaque, is visible on both planes (arrows; arrowheads indicate the edges of the plaque ulceration). ECA indicates external carotid artery.

ment with antihypertensive medication. "Diabetes mellitus" was defined as a fasting serum glucose level of $>6.9 \mathrm{mmol} / \mathrm{L}$, 2-hour postload glucose level of $>11.0 \mathrm{mmol} / \mathrm{L}$, or the use of antidiabetic medication. We assessed smoking status at the time of the TIA or ischemic stroke and dichotomized it into current smoker or no current smoker. In addition, we recorded body mass index, the use of cardiovascular medications, and medical history.

\section{MDCTA Data Acquisition and Analysis}

We performed image acquisition by using a standardized protocol, as discussed in the study design article. ${ }^{18}$ All MDCTA studies were evaluated by trained readers blinded to clinical data and other imaging tests. The MDCTA images were transferred to a workstation equipped with dedicated 3D analysis software (Leonardo and syngo.via; Siemens, Erlangen, Germany). The multiplanar reformatting application allowed analysis of both carotid arteries in oblique, coronal, and sagittal planes. Image quality was rated on a 3-point scale: 1) poor, defined as low contrast and major artifacts and not eligible for analysis; 2) moderate, defined as moderate artifacts and eligible for analysis; and 3) good, defined as few or no artifacts and eligible for analysis.

First, we evaluated the presence of an atherosclerotic plaque in both carotid arteries, defined as the presence of calcifications and/or thickening of the vessel wall ( $\geq \sim 1 \mathrm{~mm}$ ). If present, disruption of the plaque surface was assessed in both arteries at the same time. "Disruption of the plaque surface," defined as the presence of plaque ulceration and/or a fissured fibrous cap, was assessed by 2 independent observers (B.H., 12 months, and A.C.v.D., 4 years of experience); discrepancies were solved by consensus and/or an experienced third observer (A.v.d.L, 10 years of experience). We defined "plaque ulceration" as an extension of contrast material of $>1 \mathrm{~mm}$ into the atherosclerotic plaque on at least 2 orthogonal planes (Fig 1). ${ }^{13,20}$ We defined "fissured fibrous cap" according to the criteria of Saba and Mallarini, ${ }^{21}$ extension of contrast material of $<1 \mathrm{~mm}$ into the atherosclerotic plaque and an angle of $\geq 230^{\circ}$ with the lumen (Fig 2).

Interobserver variability for the initial review was moderate ( $\kappa=0.41)$ for the detection of disruption of the plaque surface (plaque ulceration and/or fissured fibrous cap), moderate $(\kappa=$ $0.46)$ for the detection of plaque ulceration, and fair $(\kappa=0.24)$ for the detection of fissured fibrous cap. Moreover, the most severe 


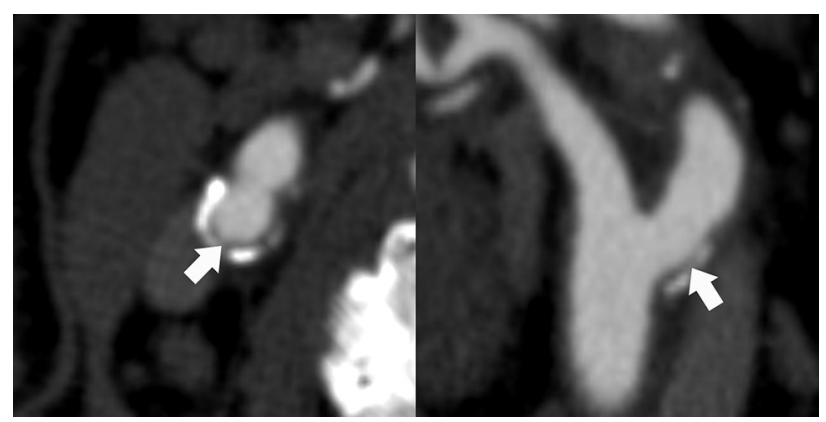

FIG 2. Fissured fibrous cap on MDCTA. Transversal (left) and longitudinal (right) MDCTA images of the carotid bifurcation. A "fissured fibrous cap," defined as an extension of contrast material of $<1 \mathrm{~mm}$ into the atherosclerotic plaque and an angle of $\geq 230^{\circ}$ with the lumen, is visible only on the transversal plane (arrows).

stenosis in the carotid bifurcations and internal carotid arteries was measured according to the ECST and NASCET criteria, perpendicular to the central lumen line. ${ }^{19,22}$ A custom-made plug-in for the freely available ImageJ software (National Institutes of Health, Bethesda, Maryland) was used to quantify calcifications in both carotid arteries within $3 \mathrm{~cm}$ proximal and distal to the bifurcation. We used a threshold of $600 \mathrm{HU}$ to differentiate calcifications from contrast material in the lumen; calcification volume was expressed in cubic millimeters. A detailed description of the measurements is provided elsewhere. ${ }^{23}$

\section{MR Imaging Data Acquisition and Analysis}

Image acquisition was performed on a 3T MR imaging system (Achieva; Philips Healthcare, Best, the Netherlands; or Discovery MR 750; GE Healthcare, Milwaukee, Wisconsin). A multisequence contrast-enhanced protocol was used; a detailed description of this protocol is provided in the study design article. ${ }^{18}$ For this study, we used the 3D-T1W fat suppressed spoiled gradient echo sequence (GE Healthcare) or the 2D-T1W inversion recovery turbo field echo sequence (Philips Healthcare). A 3D volume of the extracranial carotid artery (GE Healthcare) or 15 transverse adjoining sections of $2 \mathrm{~mm}$ each covering the entire plaque (Philips Healthcare) were imaged.

All MR imaging studies were evaluated by a trained reader (M.T.B.T., 4 years of experience) blinded to clinical data and other imaging tests. MR images were reviewed by using a standard DICOM viewer. Image quality was rated on a 5-point scale: 1) low SNR, limits use, arterial wall and vessel margins unidentifiable; 2 ) marginal SNR, arterial wall visible, with the substructure, lumen, and outer boundaries indistinct; 3) marginal SNR, wall structures identifiable with the lumen and outer boundaries partially obscured; 4) high SNR with minimal artifacts; vessel wall, lumen, and adventitial margins well-defined; and 5) high SNR without artifacts, wall architecture depicted in detail, lumen and adventitial boundary clearly defined. ${ }^{24}$ Intraplaque hemorrhage was scored in both arteries at the same time and was defined as a hyperintense signal in the plaque compared with the adjacent sternocleidomastoid muscle (Fig 3). ${ }^{24}$ In 47 vessels, the presence of intraplaque hemorrhage was assessed by a second independent observer (A.C.v.D, 4 years of experience; the minimum interval between MDCTA and MR imaging scores was 8 months) to assess

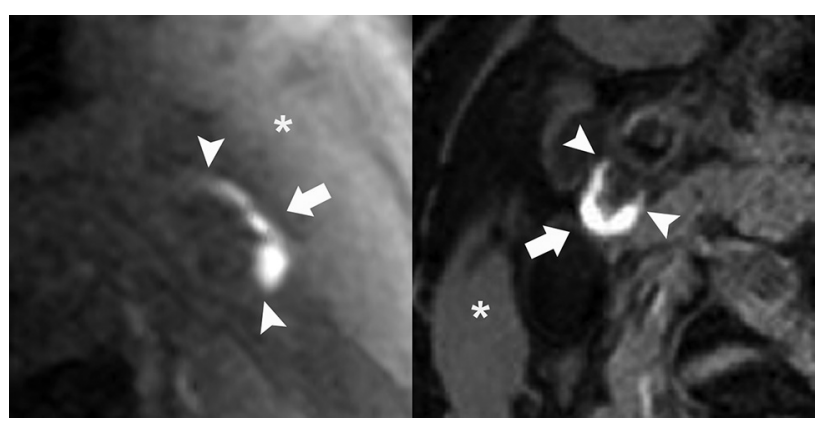

FIG 3. Intraplaque hemorrhage on MR imaging. Carotid bifurcations of 2 patients: left, the 3D-TTW fat suppressed spoiled gradient echo sequence (Discovery MR 750; GE Healthcare), and right, the 2D-TIW inversion recovery turbo field echo sequence (Achieva; Philips Healthcare). In both patients, intraplaque hemorrhage is present (arrows; arrowheads indicate the edges of the intraplaque hemorrhage), defined as a hyperintense signal in the atherosclerotic plaque compared with the sternocleidomastoid muscle (asterisk).

interobserver variability, and an excellent agreement was found $(\kappa=0.95 ; 95 \%$ CI, 0.86-1.00).

\section{Statistical Analysis}

Baseline characteristics are shown for all patients; vessel characteristics are shown for all vessels and for symptomatic and contralateral vessels separately. Data are presented as mean $\pm \mathrm{SD}$, median (25th-75th percentile), or number of patients (percentage). Differences between symptomatic and contralateral vessels were evaluated by using a $\chi^{2}$ test for categoric data and a Student $t$ test or Mann-Whitney $U$ test for continuous data. For the analysis of calcification volume, we used natural log-transformed values and added $1.0 \mathrm{~mm}^{3}$ to the nontransformed values to deal with participants with a calcification volume of zero. First, we used a $\chi^{2}$ test to assess the association between intraplaque hemorrhage and disrupted plaque surface (ulcerated plaques and/or a fissured fibrous cap) in all vessels. We used all vessels because we assumed that the underlying pathophysiologic mechanism would be similar in symptomatic and contralateral vessels. Additionally, a logistic regression was used to further investigate the association between intraplaque hemorrhage, other plaque characteristics, and cardiovascular risk factors on the one hand and disrupted plaque surface on the other. We used a generalized estimation equation approach with an unstructured correlation matrix to adjust for the correlation between both carotid arteries in each patient. Adjustments were made for age and sex (model 1) supplemented with all variables with a $P$ value $<.10$ in model 1 (model 2 , included the degree of stenosis according to the ECST criteria to correct for differences in stenosis).

Analyses were repeated to assess the association between intraplaque hemorrhage and ulcerated plaques alone. In addition, analyses were repeated for the symptomatic artery. The location of intraplaque hemorrhage and disrupted plaque surface was visually correlated by 2 independent observers after manual alignment of the MR imaging and MDCTA scans based on vessel geometry and the location of the plaque. Statistical analyses were performed by using STATA software (Version 13.1; StataCorp, College Station, Texas). $P<.05$ was considered statistically significant. 


\section{RESULTS}

\section{Patient Characteristics}

In 20 of the 100 patients, MDCTA $(n=18)$ or MR imaging $(n=$ 2 ) of the carotid arteries had not been performed due to contraindications. Two patients were excluded due to inferior image quality of the MDCTA. Of the remaining 78 patients, 149 vessels could be used for the current analyses. Seven contralateral vessels were excluded from analysis due to the absence of plaque $(n=5)$ or occlusion of the carotid artery $(n=2)$. Baseline characteristics are shown in Table 1. Forty-one of the 78 patients (53\%) had an ischemic stroke; 37 (47\%) patients had a TIA, including 7 with amaurosis fugax.

\section{Vessel Characteristics}

The median interval between the neurologic event and MDCTA was 32 days (25th to 75 th percentile, $14-56$ days); the median interval between the event and MR imaging was 44 days (25th to 75th percentile, 27-62 days). Characteristics of all vessels and symptomatic and contralateral vessels separately are shown in Table 2. The mean severity of stenosis was $51 \% \pm 17 \%$ (ECST). The prevalence of intraplaque hemorrhage in the symptomatic and contralateral vessels was $38 \%$ and $11 \%$, respectively. Twenty-

Table 1: Clinical characteristics of patients $(n=78)^{a}$

\begin{tabular}{lc}
\hline \multicolumn{1}{c}{ Clinical Characteristic } & \\
\hline Age (yr) & $67 \pm 9$ \\
Male sex & $57(73 \%)$ \\
Classification event & $30(38 \%)$ \\
TIA & $41(53 \%)$ \\
Stroke & $7(9 \%)$ \\
Amaurosis fugax & $43(55 \%)$ \\
Hypercholesterolemia & $56(72 \%)$ \\
Hypertension & $19(24 \%)$ \\
Diabetes mellitus & \\
Current smoking & $58(74 \%)$ \\
No & $20(26 \%)$ \\
Yes & $25.2(24.3-28.4)$ \\
Body mass index & \\
Current use & $36(46 \%)$ \\
Antiplatelet therapy & $0(0 \%)$ \\
Oral anticoagulants & $36(46 \%)$ \\
Statins & $50(64 \%)$ \\
Antihypertensive medication & $13(17 \%)$ \\
Antidiabetic medication & \\
History & $13(17 \%)$ \\
Ischemic stroke or TIA & $16(21 \%)$ \\
Ischemic heart disease & $15(19 \%)$ \\
Peripheral arterial disease & \\
\hline
\end{tabular}

${ }^{a}$ Data are mean \pm SD, absolute numbers of patients $(\%)$, or median (25th-75th percentile). six of the 149 vessels (17\%) showed plaque ulceration; the prevalence in the symptomatic vessels and the contralateral vessels was $27 \%$ and $7 \%$, respectively. In 13 of the 149 vessels (9\%), a fissured fibrous cap was present; most were found in the symptomatic vessels $(n=10)$, but the difference was not significant $(P=.06)$.

\section{Intraplaque Hemorrhage and Disrupted Plaque Surface}

In Table 3, the association between the presence of intraplaque hemorrhage and disrupted plaque surface (ulcerated plaque and/or fissured fibrous cap) is shown. In vessels with intraplaque hemorrhage, a disrupted plaque surface was significantly more prevalent compared with vessels without intraplaque hemorrhage (45\% versus $15 \% ; P<.001$ ). Table 4 shows the results of the multivariable logistic regression. After correction for age and sex, the presence of intraplaque hemorrhage was associated with a disrupted plaque surface (OR, 3.98; 95\% CI, 1.73-9.16; $P=.001$ ). Additionally, we found an association between the degree of stenosis and disrupted plaque surface (OR per $10 \%$ increase 1.42 ; 95\% CI, 1.09-1.84; $P=.009)$. After correction for age, sex, and all variables with $P<.10$, intraplaque hemorrhage was still significantly associated with disrupted plaque surface (OR, 3.13; 95\% CI, $1.25-7.84 ; P=.02)$. Diabetes mellitus was inversely associated with disrupted plaque surface (OR, $0.31 ; 95 \% \mathrm{CI}, 0.11-0.94 ; P=$ $.04)$. Similar results were found when the analyses were repeated to assess the association between intraplaque hemorrhage and ulcerated plaque alone. The association between intraplaque hemorrhage and disrupted plaque surface was attenuated when the analyses were repeated in only the symptomatic arteries (Online Tables 1-3). The location of the plaque ulceration and/or fissured fibrous cap was the same as that of the intraplaque hemorrhage in 16 of the 21 lesions (76\%); an example is shown in Fig 4.

Table 3: Association of intraplaque hemorrhage and disrupted plaque surface in all vessels

\begin{tabular}{lccc}
\hline & $\begin{array}{c}\text { Disrupted } \\
\text { Plaque }^{\mathrm{b}}\end{array}$ & $\begin{array}{c}\text { Intact } \\
\text { Plaque } \\
\text { Surface }\end{array}$ & Total \\
\hline $\begin{array}{l}\text { Intraplaque hemorrhage present } \\
(n=38)\end{array}$ & $17(45 \%)$ & $21(55 \%)$ & 38 \\
$\begin{array}{l}\text { Intraplaque hemorrhage absent } \\
(n=111)\end{array}$ & $17(15 \%)$ & $94(85 \%)$ & 111 \\
Total & 34 & 115 & 149 \\
\hline $\begin{array}{l}\text { a } P<.001 . \\
{ }^{b} \text { Defined as ulcerated plaque and/or fissured fibrous cap. }\end{array}$ & & \\
& & &
\end{tabular}

Table 2: Vessel characteristics ${ }^{\mathrm{a}}$

\begin{tabular}{lcccc}
\hline \multicolumn{1}{c}{ Vessel Characteristic } & All Vessels & $\begin{array}{c}\text { Symptomatic } \\
\text { Vessels }\end{array}$ & $\begin{array}{c}\text { Contralateral } \\
\text { Vessels }\end{array}$ & $\begin{array}{c}P \text { Value, Symptomatic versus } \\
\text { Contralateral Vessels }\end{array}$ \\
\hline No. & 149 & 78 & 71 & $.001^{\mathrm{b}}$ \\
Plaque ulceration & $26(17 \%)$ & $21(27 \%)$ & $5(7 \%)$ & .06 \\
Fissured fibrous cap & $13(9 \%)$ & $10(13 \%)$ & $3(4 \%)$ & .09 \\
Calcium volume (mm $\left.{ }^{3}\right)$ & $21.0(3.6-56.2)$ & $24.2(6.2-71.8)$ & $17.2(1.9-53.1)$ & $.004^{\mathrm{b}}$ \\
Degree of stenosis (ECST) (\%) & $51 \pm 17$ & $55 \pm 17$ & $47 \pm 16$ & $.03^{\mathrm{b}}$ \\
Degree of stenosis (NASCET) (\%) & $8(0-32)$ & $14(0-35)$ & $2(0-26)$ & $<.001^{\mathrm{b}}$ \\
Intraplaque hemorrhage & $38(26 \%)$ & $30(38 \%)$ & $8(11 \%)$ & \\
\hline
\end{tabular}

${ }^{a}$ Data are absolute numbers of vessels (\%), median (25th-75th percentile), or mean \pm SD.

${ }^{\mathrm{b}} \mathrm{P}<.05$. 
Table 4: Multivariable OR for the association among clinical characteristics, vessel characteristics, and disrupted plaque surface in all vessels

\begin{tabular}{|c|c|c|c|c|}
\hline \multirow[b]{2}{*}{ Characteristic } & \multicolumn{2}{|c|}{ Multivariable (Age, Sex) } & \multicolumn{2}{|c|}{$\begin{array}{c}\text { Multivariable }{ }^{a}(\text { Age, Sex, } \\
\text { Factors } P<.10)\end{array}$} \\
\hline & OR $(95 \% \mathrm{CI})$ & $P$ Value & OR $(95 \% \mathrm{Cl})$ & $P$ Value \\
\hline Age & $1.05(1.00-1.10)$ & .07 & $1.05(1.00-1.10)$ & .07 \\
\hline Sex & $0.42(0.15-1.12)$ & .08 & $0.50(0.17-1.42)$ & .19 \\
\hline Hypertension & $1.98(0.73-5.35)$ & .18 & & \\
\hline Diabetes mellitus & $0.40(0.14-1.10)$ & .08 & $0.31(0.11-0.94)$ & $.04^{\mathrm{b}}$ \\
\hline Hypercholesterolemia & $1.41(0.63-3.15)$ & .40 & & \\
\hline Current smoking & $0.48(0.16-1.46)$ & .20 & & \\
\hline Intraplaque hemorrhage & $3.98(1.73-9.16)$ & $.001^{\mathrm{b}}$ & $3.13(1.25-7.84)$ & $.02^{\mathrm{b}}$ \\
\hline Degree of stenosis (ECST, per 10\%) & $1.42(1.09-1.84)$ & $.009^{b}$ & $1.30(1.00-1.69)$ & .07 \\
\hline Calcification volume & $0.86(0.69-1.08)$ & .21 & & \\
\hline
\end{tabular}

${ }^{a}$ We corrected for age, sex, diabetes mellitus, and degree of stenosis (ECST).

${ }^{\mathrm{b}} \mathrm{p}<.05$.

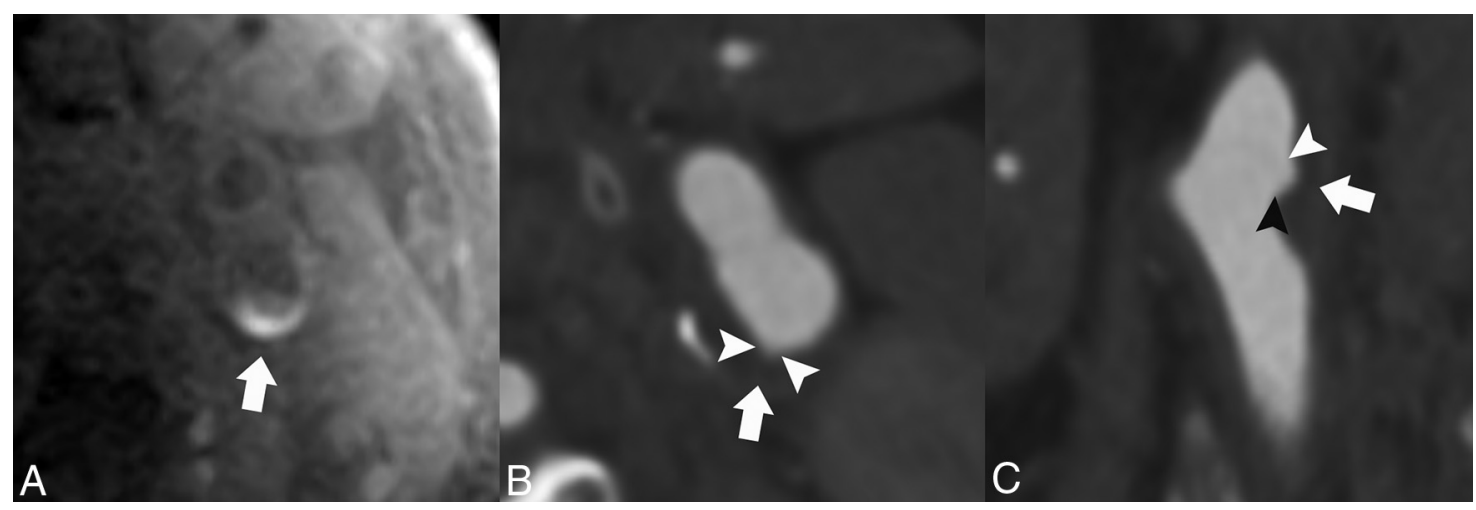

FIG 4. Correlated intraplaque hemorrhage on MR imaging and plaque ulceration on MDCTA. An example of MR imaging and MDCTA images of the left carotid bifurcation. On the 3D-TIW fat suppressed echo-spoiled gradient echo MR imaging sequence, intraplaque hemorrhage is visible in the atherosclerotic plaque (A, arrow). MDCTA images show a small ulceration on both the transversal and longitudinal plane at the site of the intraplaque hemorrhage ( $B$ and $C$, arrows; arrowheads indicate the edges of the plaque ulceration).

\section{DISCUSSION}

This study shows that intraplaque hemorrhage is associated with a disrupted plaque surface (plaque ulceration and/or fissured fibrous cap) and plaque ulceration alone. In $76 \%$ of the vessels with both intraplaque hemorrhage and a disrupted plaque surface, intraplaque hemorrhage and plaque ulceration or fissured fibrous cap shared the same location. The association between these 2 vulnerable plaque characteristics may support the notion that intraplaque hemorrhage increases the risk of plaque rupture. However, because of the cross-sectional study design, serial studies are needed to further investigate the role of intraplaque hemorrhage in plaque rupture.

Some methodologic issues need to be discussed first. A strength of our prospective study is that most vessels showed a mild stenosis. Most previous studies focused on patients with a moderate or severe carotid artery stenosis. However, plaque imaging in this patient group is less relevant for clinical decisionmaking because patients with a moderate (male patients) or severe (all patients) symptomatic carotid artery stenosis are already eligible for carotid endarterectomy. ${ }^{16}$ Moreover, the amount of calcification will probably be higher in patients with a moderate or severe carotid artery stenosis, complicating the detection of plaque surface morphology. Second, we reviewed MDCTA and MR images of all patients with a disrupted plaque surface and intraplaque hemorrhage to investigate whether the intraplaque hemorrhage and plaque disruption were at the same location.
The finding that the location was indeed similar in $76 \%$ strengthens the association between the 2 vulnerable plaque characteristics.

A limitation of our multicenter study is that we used different MDCTA and MR imaging scanners. MDCTA scanning protocols, however, were similar. We used the 3D-T1W fat suppresssed spoiled gradient echo or the $2 \mathrm{D}-\mathrm{T} 1 \mathrm{~W}$ inversion recovery turbo field echo MR imaging sequence to detect intraplaque hemorrhage. Bitar et $\mathrm{al}^{25}$ and Cappendijk et $\mathrm{al}^{26}$ used sequences like ours and found a good sensitivity, specificity, and interobserver agreement for the detection of intraplaque hemorrhage in both sequences. They also found good agreement between MR imagingdepicted intraplaque hemorrhage and histologic sections. In addition, our overall prevalence of intraplaque hemorrhage of $26 \%$ is in accordance with that in the literature. ${ }^{4,5,15}$ Correction for MR imaging protocol used in the multivariate model did not change our results. A second limitation is the lack of histology. We used MDCTA instead of MR imaging for the evaluation of the plaque surface, because MDCTA has been shown to have a high sensitivity and specificity for the detection of plaque ulceration and has fewer limitations such as partial volume effects and flow artifacts that mimic plaques. ${ }^{27,28}$ In addition, when we reviewed the MR images and MDCTA images side by side, plaque ulcerations were hardly visible on the MR images. Saba et $\mathrm{al}^{27}$ showed a good sensitivity and specificity for the detection of plaque ulceration by using MDCTA and multiplanar reconstruction (sensitivity, 
$75.8 \%$; specificity, 90.8\%). We found an overall prevalence of plaque ulceration of $17 \%$. Similar prevalence of plaque ulceration was found in other studies, ranging from $13 \%$ to $22 \%{ }^{15,20,29,30}$ Detection of the presence of a fissured fibrous cap has been studied less intensively. We found an overall prevalence of the presence of a fissured fibrous cap of $9 \%$, in agreement with the $11 \%$ found by Saba and Mallarini. ${ }^{20}$ Rupture of the fissured fibrous cap was significantly associated with cerebrovascular symptoms $(P=$ $.003) .{ }^{20}$ Based on the good sensitivity and specificity of MDCTA for plaque ulcerations and the good agreement between MR imaging-depicted intraplaque hemorrhage and histology, we therefore conclude that it is legitimate to use imaging techniques such as MDCTA and MR imaging to study the relationship between intraplaque hemorrhage and the disruption of the plaque surface. ${ }^{25,26}$ Moreover, the use of MDCTA and MR imaging provides the opportunity to investigate the relationship in a specific category of patients - that is, those with a mild-to-moderate carotid artery stenosis, patients who are treated medically and thus have no available histology. The final limitation of our study is that it is cross-sectional. Our results should therefore be confirmed in a serial study.

Rupture of an atherosclerotic carotid artery plaque is an important cause of ischemic stroke. Therefore, understanding the pathophysiology of plaque rupture is very important. Infiltration of macrophages in pathologic intima thickening plays a key role in the development of atherosclerotic plaques. The combination of macrophage infiltration, apoptosis, and hypoxia-induced necrosis leads to the development of more advanced atherosclerotic plaques with a lipid-rich necrotic core. Previous studies already showed an association between lipid-rich necrotic core volumes and plaque ulceration. ${ }^{29-31}$ In these larger plaques, hypoxia and the inflammatory response are assumed to promote neovascularization. As stated previously, the common viewpoint is that these small leaky neovessels are responsible for the occurrence of intraplaque hemorrhage and subsequently the development of an unstable rupture-prone plaque. ${ }^{5,9-12,32}$ Our results-if confirmed in serial studies_can support the pathophysiologic relation between intraplaque hemorrhage and disrupted plaque surface. An alternative viewpoint to explain this relationship is that repeated fissuring of the plaque and associated formation of nonocclusive luminal thrombus incorporated in the plaque could be the cause of intraplaque hemorrhage. ${ }^{5}$ Nevertheless, observations that intraplaque hemorrhage is related to high attenuation of plaque neovessels, in the absence of plaque fissuring, supports the more common view of small leaky neovessels as the cause of intraplaque hemorrhage. ${ }^{5,10}$

\section{CONCLUSIONS}

We showed that intraplaque hemorrhage is associated with a disrupted plaque surface (plaque ulceration and/or fissured fibrous cap) in patients with a $<70 \%$ carotid artery stenosis. Our findings suggest a strong association between these 2 vulnerable plaque characteristics. Nevertheless, because of the cross-sectional study design, additional serial studies are needed to evaluate whether intraplaque hemorrhage indeed increases the risk for plaque rupture and subsequent TIA or ischemic stroke.

\section{APPENDIX}

Participating centers: Academic Medical Center, Amsterdam (P.J. Nederkoorn); Atrium Medisch Centrum, Heerlen (A.H.C.M.L. Schreuder); Erasmus Medical Center, Rotterdam (A. van der Lugt, P.J. Koudstaal); Flevoziekenhuis, Almere (M. Limburg); Kennemer Gasthuis, Haarlem (M. Weisfelt); Laurentius Ziekenhuis, Roermond (A.G. Korten); Maasstad Ziekenhuis, Rotterdam (R. Saxena); Maastricht University Medical Center (M.E. Kooi, R.J. van Oostenbrugge, W.H. Mess); Orbis Medisch Centrum, Sittard (N.P. van Orshoven); Sint Antonius Ziekenhuis, Nieuwegein (S.C. Tromp); Sint Franciscus Gasthuis, Rotterdam (S.L.M. Bakker); Slotervaartziekenhuis, Amsterdam (N.D. Kruyt); Tergooi Ziekenhuizen, Hilversum/Blaricum (J.R. de Kruijk); University Medical Center Utrecht (J. Hendrikse, G.J. de Borst); Viecuri Medisch Centrum, Venlo (B.J. Meems); Vlietland Ziekenhuis, Schiedam (J.C.B. Verhey); IJsselland Ziekenhuis, Capelle a/d IJsel (A.D. Wijnhoud).

Disclosures: Anouk C. van Dijk—RELATED: Grant: Center for Translational Molecular Medicine and supported by the Dutch Heart Foundation, ${ }^{*}$ Comments: Project PARISK (grant 01C-202). Antonius F.W. van der Steen—RELATED: Grant: Center for Translational Molecular Medicine, project PARISK, ${ }^{*}$ Comments: This work is part of a public-private partnership by the Center for Translational Molecular Medicine and the Dutch Heart Foundation; I am Co-Principal Investigator of this grant. Mat J.A.P. Daemen-RELATED: Grant: Center for Translational Molecular Medicine, ${ }^{*}$ Comments: The Center for Translational Molecular Medicine provided the grant for the PARISK research program. Peter J. Koudstaal—UNRELATED: editor and author, Comments: Textbook of Neurology, Reed Elsevier. Paul J. Nederkoorn-UNRELATED: Grants/Grants Pending: 1) Preventive Antibiotics in Stroke Study, the Netherlands Organization for Health Research and Development (no. 171002302) and the Netherlands Heart Foundation (no. 2009B095); 2) The ThRombolysis in UnconTrolled Hypertension study: Fonds NutsOhra, grant No. 1302-026. Marianne Eline KooiRELATED: Grant: Center for Translational Molecular Medicine.* Aad van der LugtRELATED: Grant: Center for Translational Molecular Medicine, * Dutch Heart Foundation*; UNRELATED: Grants/Grants Pending: GE Healthcare, ${ }^{*}$ Comments: MRI vendor; Payment for Lectures (including service on Speakers Bureaus): GE Healthcare, ${ }^{\star}$ Comments: MRI vendor. ${ }^{*}$ Money paid to the institution.

\section{REFERENCES}

1. Yuan C, Mitsumori LM, Beach KW, et al. Carotid atherosclerotic plaque: noninvasive MR characterization and identification of vulnerable lesions. Radiology 2001;221:285-99

2. Naghavi M, Libby P, Falk E, et al. From vulnerable plaque to vulnerable patient: a call for new definitions and risk assessment strategies, part II. Circulation 2003;108:1772-78

3. Naghavi M, Libby P, Falk E, et al. From vulnerable plaque to vulnerable patient: a call for new definitions and risk assessment strategies, part I. Circulation 2003;108:1664-72

4. Saam T, Hetterich H, Hoffmann V, et al. Meta-analysis and systematic review of the predictive value of carotid plaque hemorrhage on cerebrovascular events by magnetic resonance imaging. J Am Coll Cardiol 2013;62:1081-91

5. Teng Z, Sadat U, Brown AJ, et al. Plaque hemorrhage in carotid artery disease: pathogenesis, clinical and biomechanical considerations. J Biomech 2014;47:847-58

6. Altaf N, Daniels L, Morgan PS, et al. Detection of intraplaque hemorrhage by magnetic resonance imaging in symptomatic patients with mild to moderate carotid stenosis predicts recurrent neurological events. J Vasc Surg 2008;47:337-42

7. Kwee RM, van Oostenbrugge RJ, Mess WH, et al. MRI of carotid atherosclerosis to identify TIA and stroke patients who are at risk of a recurrence. J Magn Reson Imaging 2013;37:1189-94

8. Takaya N, Yuan C, Chu B, et al. Association between carotid plaque characteristics and subsequent ischemic cerebrovascular events: a prospective assessment with MRI-initial results. Stroke 2006; $37: 818-23$ 
9. Michel JB, Virmani R, Arbustini E, et al. Intraplaque haemorrhages as the trigger of plaque vulnerability. Eur Heart J 2011;32:1977-85, $85 \mathrm{a}, 85 \mathrm{~b}, 85 \mathrm{c}$

10. Virmani R, Kolodgie FD, Burke AP, et al. Atherosclerotic plaque progression and vulnerability to rupture: angiogenesis as a source of intraplaque hemorrhage. Arterioscler Thromb Vasc Biol 2005; 25:2054-61

11. Michel JB, Delbosc S, Ho-Tin-Noé B, et al. From intraplaque haemorrhages to plaque vulnerability: biological consequences of intraplaque haemorrhages. J Cardiovasc Med (Hagerstown) 2012;13: $628-34$

12. Takaya N, Yuan C, Chu B, et al. Presence of intraplaque hemorrhage stimulates progression of carotid atherosclerotic plaques: a highresolution magnetic resonance imaging study. Circulation 2005; 111:2768-75

13. Lovett JK, Gallagher PJ, Hands LJ, et al. Histological correlates of carotid plaque surface morphology on lumen contrast imaging. Circulation 2004;110:2190-97

14. Saba L, Sanfilippo R, Pirisi R, et al. Multidetector-row CT angiography in the study of atherosclerotic carotid arteries. Neuroradiology 2007;49:623-37

15. U-King-Im JM, Fox AJ, Aviv RI, et al. Characterization of carotid plaque hemorrhage: a CT angiography and MR intraplaque hemorrhage study. Stroke 2010;41:1623-29

16. Rothwell PM, Eliasziw M, Gutnikov SA, et al; Carotid Endarterectomy Trialists Collaboration. Endarterectomy for symptomatic carotid stenosis in relation to clinical subgroups and timing of surgery. Lancet 2004;363:915-24

17. Rothwell PM, Gibson R, Warlow CP; European Carotid Surgery Trialists' Collaborative Group. Interrelation between plaque surface morphology and degree of stenosis on carotid angiograms and the risk of ischemic stroke in patients with symptomatic carotid stenosis. Stroke 2000;31:615-21

18. Truijman MT, Kooi ME, van Dijk AC, et al. Plaque At RISK (PARISK): prospective multicenter study to improve diagnosis of high-risk carotid plaques. Int J Stroke 2014;9:747-54

19. Randomised trial of endarterectomy for recently symptomatic carotid stenosis: final results of the MRC European Carotid Surgery Trial (ECST). Lancet 1998;351:1379-87

20. de Weert TT, Cretier S, Groen HC, et al. Atherosclerotic plaque surface morphology in the carotid bifurcation assessed with multidetector computed tomography angiography. Stroke 2009;40:1334-40
21. Saba L, Mallarini G. Fissured fibrous cap of vulnerable carotid plaques and symptomaticity: are they correlated? Preliminary results by using multi-detector-row CT angiography. Cerebrovasc Dis 2009;27:322-27

22. North American Symptomatic Carotid Endarterectomy Trial Collaborators. Beneficial effect of carotid endarterectomy in symptomatic patients with high-grade carotid stenosis. $\mathrm{N} \mathrm{Engl} \mathrm{J} \mathrm{Med}$ 1991;325:445-53

23. de Weert TT, Cakir H, Rozie S, et al. Intracranial internal carotid artery calcifications: association with vascular risk factors and ischemic cerebrovascular disease. AJNR Am J Neuroradiol 2009;30:177-84

24. Yuan C, Mitsumori LM, Ferguson MS, et al. In vivo accuracy of multispectral magnetic resonance imaging for identifying lipid-rich necrotic cores and intraplaque hemorrhage in advanced human carotid plaques. Circulation 2001;104:2051-56

25. Bitar R, Moody AR, Leung G, et al. In vivo 3D high-spatial-resolution MR imaging of intraplaque hemorrhage. Radiology 2008;249:259-67

26. Cappendijk VC, Cleutjens KB, Heeneman S, et al. In vivo detection of hemorrhage in human atherosclerotic plaques with magnetic resonance imaging. J Magn Reson Imaging 2004;20:105-10

27. Saba L, Caddeo G, Sanfilippo R, et al. Efficacy and sensitivity of axial scans and different reconstruction methods in the study of the ulcerated carotid plaque using multidetector-row CT angiography: comparison with surgical results. AJNR Am J Neuroradiol 2007; 28:716-23

28. Yu W, Underhill HR, Ferguson MS, et al. The added value of longitudinal black-blood cardiovascular magnetic resonance angiography in the cross sectional identification of carotid atherosclerotic ulceration. J Cardiovasc Magn Reson 2009;11:31

29. Homburg PJ, Rozie S, van Gils MJ, et al. Association between carotid artery plaque ulceration and plaque composition evaluated with multidetector CT angiography. Stroke 2011;42:367-72

30. Saba L, Sanfilippo R, Sannia S, et al. Association between carotid artery plaque volume, composition, and ulceration: a retrospective assessment with MDCT. AJR Am J Roentgenol 2012;199:151-56

31. Underhill HR, Yuan C, Yarnykh VL, et al. Predictors of surface disruption with $M R$ imaging in asymptomatic carotid artery stenosis. AJNR Am J Neuroradiol 2010;31:487-93

32. DeMarco JK, Huston J 3rd. Imaging of high-risk carotid artery plaques: current status and future directions. Neurosurg Focus 2014; 36:E1 